Check for updates

Cite this: RSC Adv., 2017, 7, 22215

\title{
A label-free detection of diethylstilbestrol based on molecularly imprinted polymer-coated upconversion nanoparticles obtained by surface grafting $\dagger$
}

\author{
Yu Wang, ${ }^{a}$ Shuyue Ren, ${ }^{a}$ Huicong Jiang, ${ }^{\text {ab }}$ Yuan Peng, ${ }^{a}$ Jialei Bai, ${ }^{a}$ Qiaofeng Li, ${ }^{\text {ab }}$ \\ Chao Li, ${ }^{a}$ Zhixian Gao*a and Baoan Ning ${ }^{\star a}$
}

Herein, we report a novel fluorescent sensor based on molecularly imprinted polymer-coated upconversion nanoparticles (MIP-coated UCNPS) for the recognition and detection of diethylstilbestrol (DES). We used one-step process to modify the surface of the UCNPs with alkenyl groups, which was not only a simple process, but also greatly saved the preparation time. Then, a molecularly imprinted polymer was synthesized on the UCNPs by the surface-graft molecular imprinting method. MIP-coated UCNPs combine the advantages of UCNPs and molecularly imprinted polymers such as strong fluorescence properties, excellent stability, reusability, good adsorption capacity, and high specificity. After the removal of the template molecules, the UCNPs were able to selectively recognize DES, and the fluorescence intensity decreased as the concentration of DES increased. A good linear relationship was obtained in the concentration range of $50-1000 \mathrm{ng} \mathrm{mL}^{-1}$ with the correlation coefficient of 0.9989 , and the detection limit was $12.8 \mathrm{ng} \mathrm{mL}^{-1}(\mathrm{~S} / \mathrm{N}=3)$. MIP-coated UCNPs could be applied for the detection of DES in real milk samples, and the average recoveries for five different concentrations ranged from $90.3 \%$ to $107.8 \%$, with the relative standard deviations (RSD) below $2.7 \%$. This fluorescence sensor provides a more convenient, simple and highly specific method for the on-field detection of DES and is promising for a wide range of applications in the future.

Received 18th November 2016 Accepted 29th January 2017

DOI: $10.1039 / c 6 r a 26999 k$

rsc.li/rsc-advances are sensitive and accurate, but they require complicated sample treatment and are time-consuming. Colloidal-gold strip ${ }^{\mathbf{1 5 , 1 6}}$ is fast and easy to operate, but the immune antibody is difficult to produce and may lose biological activity upon external treatment. With the emergence and development of new technologies, sensors have become a hot topic of research because of their fast response and efficiency.

In the field of sensors, upconversion nanoparticles (UCNPs) and molecularly imprinted polymers (MIPs) are of great interest. Moreover, the rare earth element-doped UCNPs are widely used in biosensing technology. ${ }^{17}$ The special fluorescence mechanism of UCNPs makes them a superior nanobiolabeled material. ${ }^{18}$ Compared to other common fluorescent probes, in particular, quantum dots (QDs), ${ }^{19,20}$ UCNPs present significant advantages such as high luminescence, no toxicity towards organisms, narrow absorption and emission peaks, long lifetime, and virtually zero interference background..$^{21}$ MIPs, as an affinity medium, have the predetermined recognition ability for the imprinted template compound. ${ }^{22}$ As a highly selective solid-phase extraction material, MIPs are widely used in chromatographic separations, chemo/ biosensing, catalysis, and other fields. ${ }^{23,24}$ Moreover, compared with natural antibodies, MIPs, as an attractive material, not only have a low cost and good stability but also can be directly
${ }^{a}$ Tianjin Key Laboratory of Risk Assessment and Control Technology for Environment and Food Safety, Tianjin Institute of Health and Environment Medicine, Tianjin 84655403; Tel: +8622 84655403

${ }^{b}$ School of Public Health, Zhengzhou University, Zhengzhou 450001, P. R. China

$\dagger$ Electronic supplementary information (ESI) available: TGA curves of UCNPs and MIPs-coated UCNPs, UV absorption spectra of DES and UCNPs, chemical structures of five analogues, repeatability and stability of MIPs-coated UCNPs, effects of identification of the peak of the IR spectra, comparison between this method and other methods. See DOI: 10.1039/c6ra26999k 
synthesized. ${ }^{25}$ Based on these considerations, a label-free, sensitive, stable sensor for the on-field fast detection of DES in food was developed by combining the fluorescence characteristics of UCNPS and the selective recognition ability of MIPs.

Our research team has been studying the detection of estrogen by UCNPs and MIPs for a long time. To date, there have been a few reports on the combination of UCNPs and MIPs for sensing. ${ }^{26,27}$ In these reports, template monomers and functional monomers were combined by covalent bonding. Considering that covalent bonding between template and functional monomer can cause difficulty in target removing, which leads to lower adsorption capacity, we fabricated MIPs via non-covalent bonding and prepared MIPs-coated UCNPs for the fluorescence sensing of DES. The detection method based on this sensor has low toxicity and a good reproducibility, but the modification of UCNPs is a bit complex and the detection range is narrow. Therefore, it needs further improvement.

In this study, we modified the surface of UCNPs with alkenyl groups by a one-step method, which was not only simple, but also greatly saved preparation time. The imprinted layer on the surface of the UCNPs was synthesized by the surface graft molecularly imprinting method. The imprinted layer could selectively and sensitively recognize the target, and experiments showed that the polymers retained the properties of stability and repeatability. Moreover, the adsorption capacity of this material was adequate, and the adsorption rate was relatively fast, such that in $40 \mathrm{~min}$, an adsorption equilibrium could be achieved. This unique sensor was also demonstrated to be an efficient and easy to operate system for the selective detection of DES in milk.

\section{Experimental}

\section{Reagents}

All the reagents used herein were of at least analytical grade. $\mathrm{YbCl}_{3} \cdot 6 \mathrm{H}_{2} \mathrm{O}$ (99.99\%), $\mathrm{YCl}_{3} \cdot 7 \mathrm{H}_{2} \mathrm{O}(99.99 \%), \mathrm{ErCl}_{3} \cdot 6 \mathrm{H}_{2} \mathrm{O}(99.9 \%)$, 1-octadecene (ODE), tetraethoxysilane (TEOS), and 3-(methacryloyloxy)propyl] trimethoxysilane (MPS) were purchased from Sigma-Aldrich (Shanghai, China). Oleic acid (OA), methacrylic acid (MAA), $\mathrm{NaOH}$, and $\mathrm{NH}_{4} \mathrm{~F}$ were purchased from Xiya Chemical Reagent (Shandong, China). Ethanol, methanol, glacial acetic acid (HAc), cyclohexane, ammonia (25\%), acetonitrile, and toluene were purchased from Tianjin Fuyu Co. Ltd. (Tianjin, China). Ethylene glycol dimethacrylate (EGDMA), 2,2'-azobisisobutyronitrile (AIBN) (2,2'-azobis(2-methylpropionitrile)), diethylstilbestrol (DES), estradiol $\left(\mathrm{E}_{2}\right)$, hexestrol (HEX), dienestrol (DIEN), and bisphenol A (BPA) were purchased from J\&K Chemical Ltd. (Beijing, China). Millipore Milli-Q ultrapure water (Millipore, 18.2 M $\Omega \mathrm{cm}$ ) was prepared in our laboratory.

\section{Apparatus}

Ultraviolet-visible (UV-vis) spectra were obtained over 200$500 \mathrm{~nm}$ using a TU-1901 UV-vis spectrophotometer (Beijing Purkinje General Instrument Co. Ltd). X-ray diffraction (XRD) patterns were obtained using a D/max-2500 diffractometer (Rigaku, Japan). The morphology of the prepared samples was investigated by transmission electron microscopy (JEM2010FEF, JEOL, Japan). Fourier transform infrared (FT-IR) spectra (4000-400 $\mathrm{cm}^{-1}$ ) were obtained by a FTS6000 spectrometer (Bio-rad, America) using $\mathrm{KBr}$ pellets. Fluorescence measurements were performed using an F-4500 fluorescence spectrophotometer (Hitachi, Japan) with an external $980 \mathrm{~nm}$ laser diode (Hi-TechOptoelectronic Co. Ltd, China) as the excitation source. Thermogravimetric analysis (TGA) was performed using a TG209 DSC204 DMA242 TMA202 (NETZSCH, Germany).

\section{Synthesis of $\mathrm{NaYF}_{4}: \mathrm{Yb}, \mathrm{Er}$ UCNPs}

UCNPs were synthesized according to a previously reported method with a modified procedure. ${ }^{28} \mathrm{YbCl}_{3} \cdot 6 \mathrm{H}_{2} \mathrm{O}(0.18 \mathrm{mmol})$, $\mathrm{YCl}_{3} \cdot 7 \mathrm{H}_{2} \mathrm{O}(0.80 \mathrm{mmol})$, and $\mathrm{ErCl}_{3} \cdot 6 \mathrm{H}_{2} \mathrm{O}(0.02 \mathrm{mmol})$ were mixed with $10 \mathrm{~mL}$ oleic acid (OA) and $30 \mathrm{~mL}$ 1-octadecene (ODE) in a $100 \mathrm{~mL}$ flask. The mixture was heated to $160{ }^{\circ} \mathrm{C}$ for $30 \mathrm{~min}$ to form a uniform solution, oxygen and water were removed, and then the solution was cooled down to $50{ }^{\circ} \mathrm{C}$. To this solution, $20 \mathrm{~mL}$ of a methanol solution containing $\mathrm{NH}_{4} \mathrm{~F}(8 \mathrm{mmol})$ and $\mathrm{NaOH}$ ( $5 \mathrm{mmol}$ ) was added dropwise and stirred at $50{ }^{\circ} \mathrm{C}$ for $1 \mathrm{~h}$. The solution was heated to $100{ }^{\circ} \mathrm{C}$ for $20 \mathrm{~min}$ to distill methanol, fast-heated to $320{ }^{\circ} \mathrm{C}$, and then maintained at this temperature for $1 \mathrm{~h}$ under a $\mathrm{N}_{2}$ atmosphere. When reaction was complete, the resultant solution was allowed to naturally cool down and the UCNPs were obtained by centrifugation. The resultant mixture was washed four times with cyclohexane/ ethanol mixture $(1: 1 \mathrm{v} / \mathrm{v})$.

\section{Silica coating of UCNPs and surface functionalization with vinyl groups by a one-step process}

MPS-UCNPs@SiO ${ }_{2}$ were synthesized on the basis of previously reported method with a modified procedure. ${ }^{29,30}$ In a $250 \mathrm{~mL}$ round bottom flask, $100 \mathrm{mg}$ of UCNPs were uniformly dispersed in $60 \mathrm{~mL}$ of ethanol. Then, $2.5 \mathrm{~mL} \mathrm{NH}{ }_{3} \cdot \mathrm{H}_{2} \mathrm{O}, 20 \mathrm{~mL}$ ultrapure water, and $25 \mu \mathrm{L}$ TEOS were added. The solution was stirred at $40{ }^{\circ} \mathrm{C}$ for $5 \mathrm{~h}$. Subsequently, $30 \mu \mathrm{L}$ HAc and $1.5 \mathrm{~mL}$ MPS were added and mixture was kept at $40{ }^{\circ} \mathrm{C}$ for $6 \mathrm{~h}$ under stirring. MPSUCNPs@SiO ${ }_{2}$ were obtained by centrifugation, and washed four times with ethanol.

\section{Synthesis of MIP-coated UCNPs}

MIP-coated UCNPs were prepared as follows. MAA (1.8 mmol) and DES $(0.3 \mathrm{mmol})$ were added to $41 \mathrm{~mL}$ acetonitrile. The mixture was slowly stirred using a magnetic stirrer for $24 \mathrm{~h}$ at room temperature to form the template-monomer complex. Modified UCNPs (60 mg), the free-radical initiator AIBN (30.0 $\mathrm{mg})$, the cross-linkers EGDMA $(3.0 \mathrm{mmol})$, and toluene $(11 \mathrm{~mL})$ were dissolved in the template-monomer solution in a $250 \mathrm{~mL}$ flask. Then, the flask was sealed under $\mathrm{N}_{2}$ and stirred under reflux in an oil bath at $45^{\circ} \mathrm{C}$ for $5 \mathrm{~h}$, then at $60^{\circ} \mathrm{C}$ for 1 day, and finally at $80{ }^{\circ} \mathrm{C}$ for $2 \mathrm{~h}$. After polymerization, MIP-coated UCNPs were obtained by centrifugation and washed several times with methanol : HAc $(9: 1, \mathrm{v} / \mathrm{v})$ until templates could not be detected by UV-vis spectrophotometry. The non-imprinted polymers (NIPs) were prepared by the same method but in the absence of the template molecule DES. 


\section{Fluorescence measurements}

Herein, the F-4500 fluorescence spectrometer (excitation power: $230 \mathrm{~mW}$; excitation and emission slits: $1 \mathrm{~nm}$ ) was used to detect all the fluorescence intensity and the emission intensity of $544 \mathrm{~nm}$ was chosen as quantitative standard. In $4 \mathrm{~mL}$ of acetonitrile, $2 \mathrm{mg}$ MIP-coated UCNPs were dispersed and completely mixed. Then, $60 \mu \mathrm{L}$ of this dispersion was added to $4.0 \mathrm{~mL}$ of acetonitrile solutions with different concentrations of DES; the fluorescence spectra for each concentration were obtained after oscillating the sample for $40 \mathrm{~min}$ at room temperature.

\section{Characterization of the MIP-coated UCNPs}

For the equilibrium binding experiments, $2 \mathrm{~mL}$ of each concentration of DES acetonitrile solution was added to $5 \mathrm{mg}$ MIP-coated UCNPs or NIP-coated UCNPs. The mixture was shaken using a mediation oscillator at room temperature for $4 \mathrm{~h}$. A UV-vis spectrophotometer was used to investigate the supernatant.

For the rebinding kinetics experiments, $10 \mathrm{mg}$ of MIP-coated UCNPs or NIP-coated UCNPs and $5 \mathrm{~mL}$ of DES acetonitrile solution with a concentration of $100 \mu \mathrm{g} \mathrm{mL}^{-1}$ were mixed in a 7 $\mathrm{mL}$ Eppendorf tube. The mixed solution was shaken for different times $(0,5,10,15,20,30,40,50$, and $60 \mathrm{~min})$ at room temperature, and the supernatant was analyzed by a UV-vis spectrophotometer.

The selectivity test was carried out by detecting DIEN, HEX, BPA, and $\mathrm{E}_{2}$ as analogues of DES at different concentrations (0, $100,200,400,600,800$, and $1000 \mathrm{ng} \mathrm{mL}^{-1}$ ). The measurements were conducted by the same method as the one detailed in "Fluorescence measurements".

\section{Detection of DES in real samples}

Milk samples used in this study were purchased from a local supermarket and were analyzed to ensure that they did not contain any DES. The pre-treatment method was based on a previous report. ${ }^{31}$ Milk $(1 \mathrm{~mL})$ was placed in a centrifuge tube. DES was added at five different concentrations: 60, 120, 240, 480 , and $960 \mathrm{ng} \mathrm{mL}{ }^{-1}$. Then, the mixture was extracted with an equal volume of ethyl acetate and left to stand until stratification. The supernatant was collected and dried under $\mathrm{N}_{2}$. Finally, the residue was redissolved in acetonitrile for analysis.

\section{Results and discussion}

\section{Preparation and characterization of MIP-coated UCNPs}

Scheme 1 illustrates the DES identification mechanism and the synthetic procedure for MIP-coated UCNPs. The molecularly imprinted polymer was formed by a simple method of polymerization on the outer surface of the UCNPs. First, UNCPs with a uniform dimension were synthesized via a solvent thermal process. Then, the surface of the UCNPs was modified with alkenyl groups by a one-step process, which greatly saved preparation time. Before polymerization, DES and the functional monomer MAA were mixed in the ratio of $1: 6$ (ref. 27) in acetonitrile for $12 \mathrm{~h}$, such that the carboxyl groups of MAA and

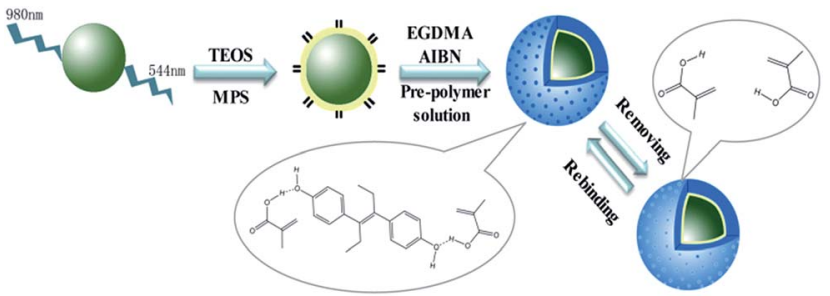

Scheme 1 Schematic of the preparation procedure for MIP-coated UCNPS.

the hydroxyl groups of DES could form the pre-polymer via hydrogen bonds. Then, the cross-linking agent (EGDMA) was added, and the polymerization occurred between the double bonds under the action of the initiator AIBN, and the imprinted polymeric layer was formed on the surface of the UCNPs. Finally, the synthesized product was eluted with methanol : HAc $(9: 1, \mathrm{v} / \mathrm{v})$ to break the hydrogen bonds between the template and the monomer and remove the template. This way, specific recognition sites were formed in the imprinted cavities.

The morphology of MIP-coated UCNPs was characterized by TEM. As it can be seen in Fig. 1a, the UCNPs were hexagonal with a uniform size (100-110 nm). Fig. 1b shows the TEM image of silica-coated UCNPs, and it could be observed that there was a thin shell $(3 \mathrm{~nm})$ on the surface of the UCNPs. In the TEM image of MIP-coated UCNPs, shown in Fig. 1c, a shell of about $8 \mathrm{~nm}$ could be clearly observed, indicating that core-shell UCNPs were obtained. Fig. S1 $\uparrow$ shows the TGA results for UCNPs and MIP-coated UCNPs. The decomposition of MIPs leads to a quick weight loss $(16 \%)$ from $350{ }^{\circ} \mathrm{C}$ to $800{ }^{\circ} \mathrm{C}$. The curves indicated that $\mathrm{H}_{2} \mathrm{O}$ and oleic acid adsorbed on the surface of UCNPs were about $4 \%$ and the amount of MIPs coating the surface was about $12 \%$. Thus, these results demonstrated that the coating procedure was successful.

The XRD patterns of UCNPs and MIP-coated UCNPs are shown in Fig. 1d. The XRD spectrum of UCNPs showed a crystalline $\beta$ phase, and it was in good agreement with the JCPDS card 28-1192. The XRD pattern of UCNPs was similar to that of MIP-coated UCNPs, thus proving that the crystalline phase of UCNPs did not change after coating with MIPs. Moreover, due to the MIP layer surface, the XRD pattern of MIP-coated UCNPs had a lower response, which demonstrated that the MIP layer was successfully coated onto the UCNPs.

To further confirm the surface modification procedures, the products were characterized by FT-IR spectrometry. Fig. 2a shows the IR spectrum of OA-UCNPs without any modification. The absorption peak at $1703 \mathrm{~cm}^{-1}$ was attributed to the stretching vibrations of the carbonyl group $(\mathrm{C}=\mathrm{O})$. The two peaks at $2926 \mathrm{~cm}^{-1}$ and $2856 \mathrm{~cm}^{-1}$ were ascribed to the methylene groups $\left(-\mathrm{CH}_{2}-\right)$ of oleic acid. In the IR spectrum of MPSUCNPs@SiO ${ }_{2}$, shown in Fig. 2b, we could see a strong peak at $1090 \mathrm{~cm}^{-1}$ and two small peaks at $808 \mathrm{~cm}^{-1}$ and $942 \mathrm{~cm}^{-1}$, corresponding to the symmetrical stretching vibration and bending vibration of the silicon-oxygen bond ( $\mathrm{Si}-\mathrm{O})$, respectively. The characteristic peaks at $1716 \mathrm{~cm}^{-1}$ and $1636 \mathrm{~cm}^{-1}$ were attributed to the stretching vibrations of the carbonyl 

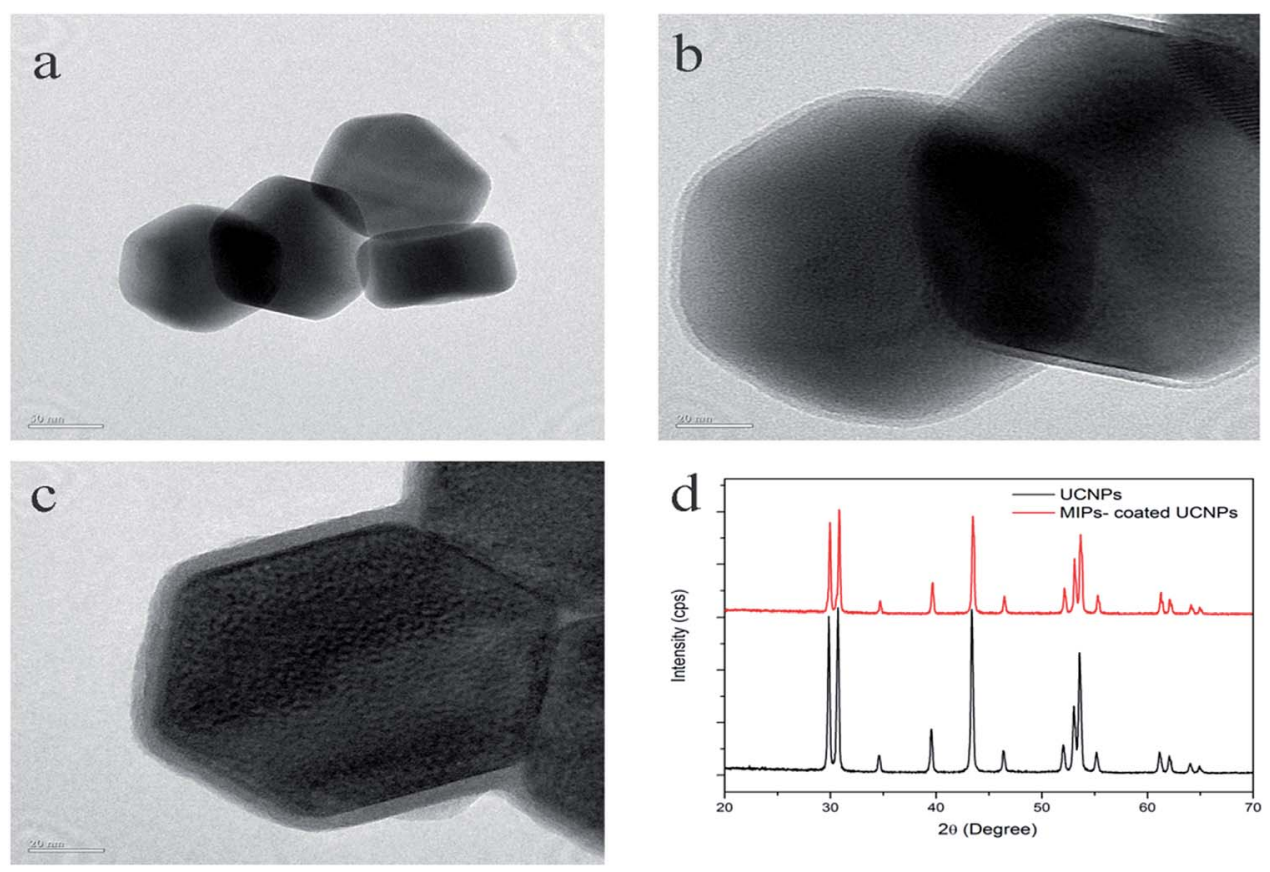

Fig. 1 TEM image of UCNPs (a), MPS-UCNPs@SiO 2 (b), and MIP-coated UCNPs (c); (d) XRD patterns of UCNPs and MIP-coated UCNPs.

group $(\mathrm{C}=\mathrm{O})$ and the carbon-carbon double bond $(\mathrm{C}=\mathrm{C})$ of MPS. Thus, it was verified that the UCNPs were successfully encapsulated by a silica shell and modified with MPS. After coating with a MIP layer, the IR spectrum, as shown in Fig. 2c, revealed two peaks at $2925 \mathrm{~cm}^{-1}$ and $2955 \mathrm{~cm}^{-1}$, corresponding to the bending vibrations of methylene $\left(-\mathrm{CH}_{2}-\right)$ and methyl groups $\left(\mathrm{CH}_{3}-\right)$, respectively. The absorption peaks at $1722 \mathrm{~cm}^{-1}$ and $1163 \mathrm{~cm}^{-1}$ were attributed to the stretching vibrations of the carbonyl group $(\mathrm{C}=\mathrm{O})$ and ether group $(\mathrm{C}-\mathrm{O}-\mathrm{C})$, respectively. The identifications of the peaks of IR spectra are described in Table S1.†

\section{Equilibrium binding}

The static adsorption experiments of the MIPs-coated UCNPs (NIPs-coated UCNPs) to DES were carried out by employing $5 \mathrm{mg}$ of

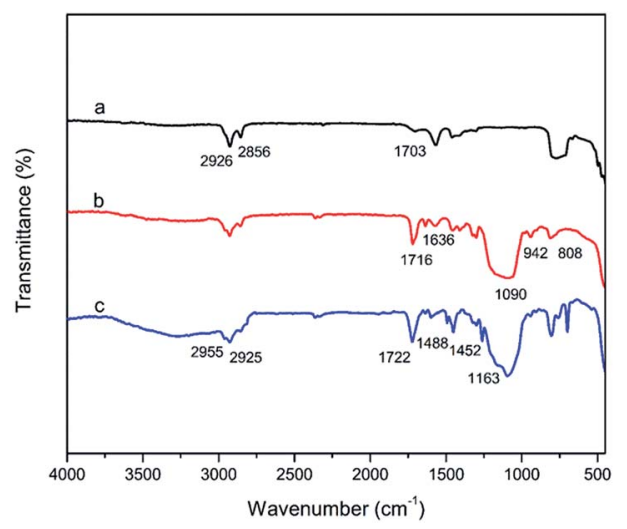

Fig. 2 FT-IR spectra of UCNPs (a), MPS-UCNPsaSiO 2 (b), and MIPcoated UCNPs (c). the MIPs-coated UCNPs (NIPs-coated UCNPs) incubated with DES acetonitrile solution $\left(10,20,40,60,80,100\right.$, and $\left.120 \mu \mathrm{g} \mathrm{mL} \mathrm{m}^{-1}\right)$ for $3 \mathrm{~h}$. Fig. 3a shows that the adsorption capacity $Q\left(\mathrm{mg} \mathrm{g}^{-1}\right)$ gradually increased as the DES concentration increased. The $Q$ of NIPcoated UCNPs was significantly lower than that of MIP-coated UCNPs. The adsorption of MIPs-coated UCNPs and NIPs-coated UCNPs tended to be saturated at the concentration of $100 \mu \mathrm{g}$ $\mathrm{mL}^{-1}$ and the adsorption capacity of MIP-coated UCNPs was significantly higher than that of NIP-coated UCNPs. The equilibrium binding isotherm was obtained by Langmuir fitting of the data. Fig. $3 \mathrm{c}$ shows the Langmuir adsorption isotherm, and the equation was $C_{\mathrm{DES}} / Q=0.000127 C_{\mathrm{DES}}+0.00354$. The maximum adsorption capacity $\left(Q_{\max }\right)$ is $7.87 \mathrm{mg} \mathrm{g}^{-1}$. The greater adsorption capacity provided a foundation to achieve a wider detection range, which avoided gradient dilutions during real sample monitoring.

To demonstrate the kinetic adsorption process of MIPcoated UCNPs and NIP-coated UCNPs, experiments were conducted by incubating $10 \mathrm{mg}$ of MIP-coated UCNPs or NIP-coated UCNPs in a DES acetonitrile solution $\left(5 \mathrm{~mL}, 100 \mu \mathrm{g} \mathrm{mL}{ }^{-1}\right)$ for different time periods. The results, as shown in Fig. $3 \mathrm{~b}$, suggested that MIP-coated UCNPs had a quick binding rate in $20 \mathrm{~min}$ and the adsorption capacity was close to the maximum value. However, we observed that for MIP-coated UCNPs, the adsorption towards DES was low within the 20 to $40 \mathrm{~min}$ period. Finally, we chose $40 \mathrm{~min}$ as the response time to ensure that MIP-coated UCNPs adsorb as much DES as possible. The adsorption rate of MIP-coated UCNPs was significantly higher than that previously reported, ${ }^{32}$ which was attributed to the surface graft molecularly imprinting method. The higher adsorption rate meant that MIP-coated UCNPs have promising applications in on-field detection. 

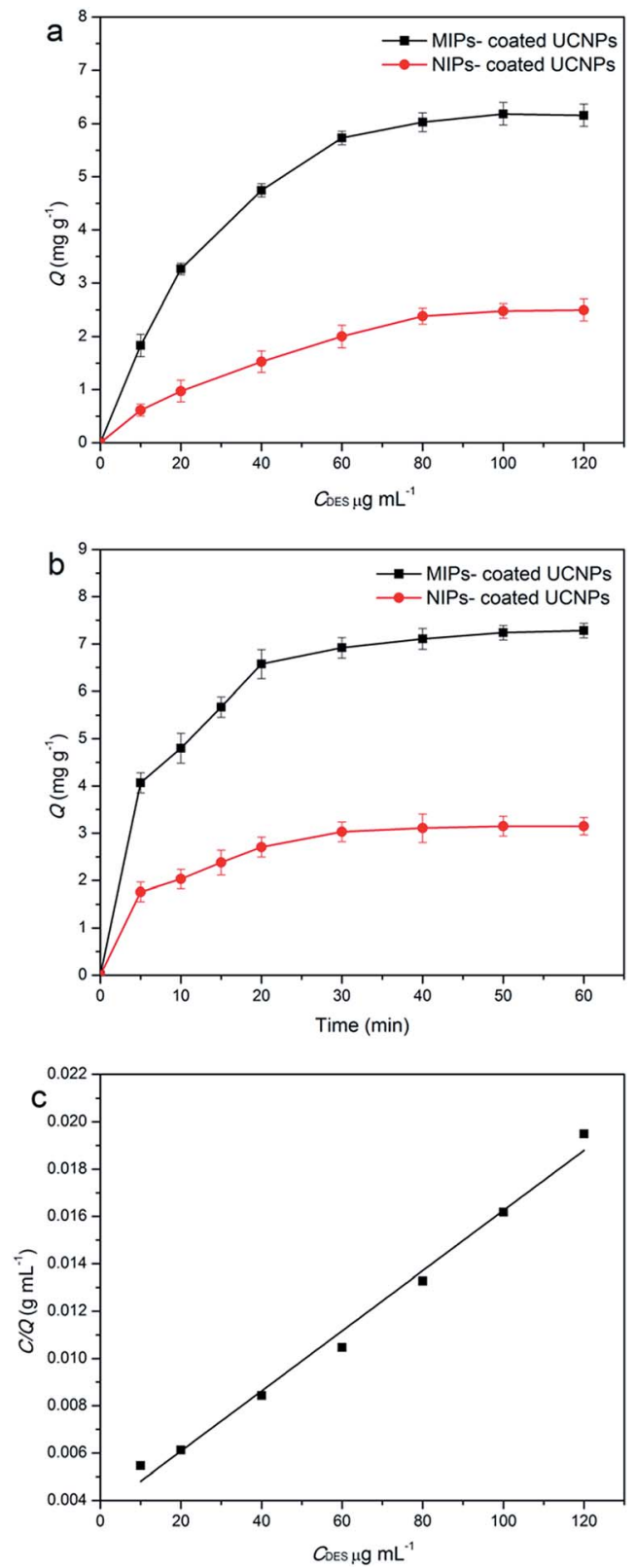

Fig. 3 (a) Adsorption isotherms of MIP-coated UCNPs and NIPcoated UCNPs at $30^{\circ} \mathrm{C}$. (b) Kinetic uptake plot of MIP-coated UCNPs and NIP-coated UCNPs at $30^{\circ} \mathrm{C}$. (c) Langmuir plot of MIP-coated UCNP $Q$ versus DES concentration.

\section{Fluorescence optosensing of DES based on MIP-coated UCNPs}

To acquire the standard curve, MIP-coated UCNPs were respectively added to a series of concentrations of DES solutions, and then the fluorescence response spectra were obtained. According to the kinetic adsorption experiment described above, $40 \mathrm{~min}$ was considered to be the optimal incubation time for MIP-coated UCNPs with DES. As shown in Fig. 4, within the DES concentration range of $50-1000 \mathrm{ng} \mathrm{mL}^{-1}$, the fluorescence intensity of MIP-coated UCNPs decreased as the concentration of DES increased, showing a good linear relationship. The quenching mechanism might be attributed to the charge transfer from UCNPs to DES. ${ }^{33}$ The DES absorption peak (Fig. S2a $\dagger$ ) and the
UCNPs emission peak (at $541 \mathrm{~nm}$ ) did not overlap. Therefore, the quenching mechanism is not through fluorescence resonance energy transfer. Upon comparing Fig. S2a with S2b, $\dagger$ it can be observed that the band gap of UCNPs was close to the absorption peak of DES. Therefore, the charge on UCNPs could be transferred to the adsorbed DES, leading to fluorescence quenching. The fluorescence quenching in this system could be described by the Stern-Volmer equation:

$$
F_{0} / F=1+K_{\mathrm{SV}}\left[\mathrm{C}_{\mathrm{q}}\right]
$$
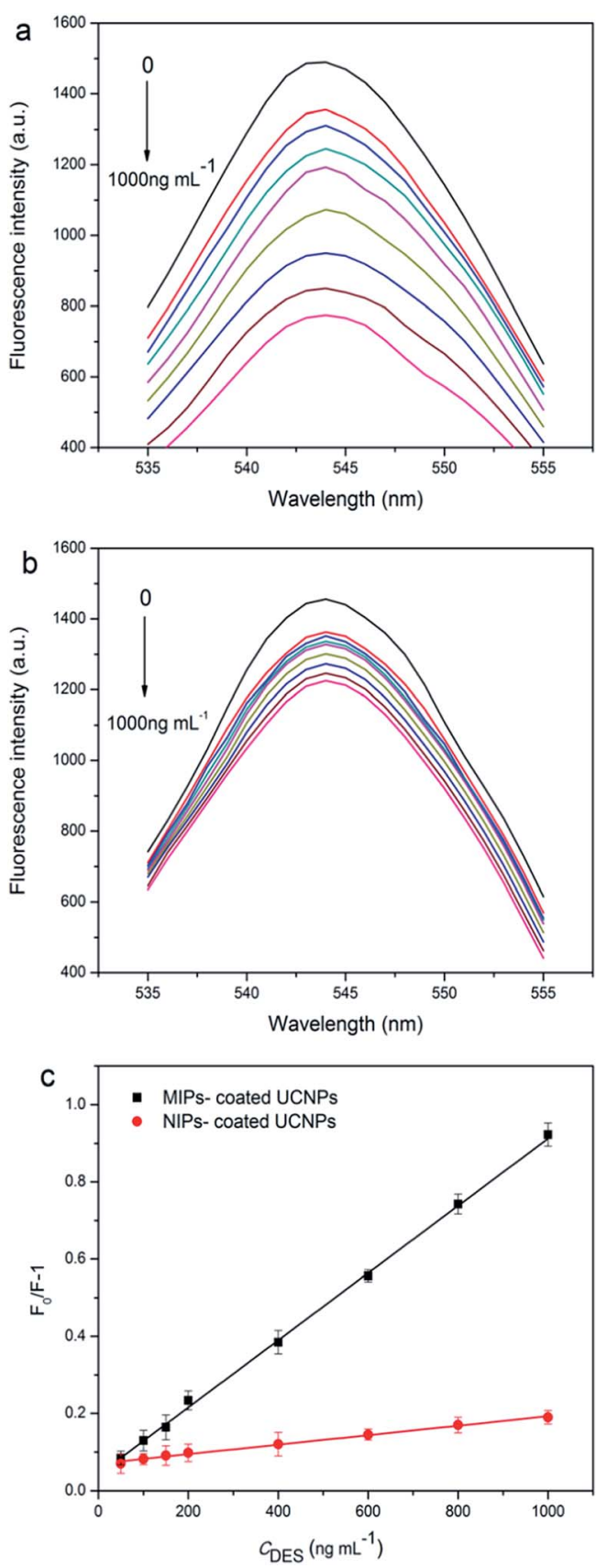

Fig. 4 Fluorescence spectra of MIP-coated UCNPs (a) and NIPcoated UCNPs (b) with different concentrations of DES; (c) SternVolmer curves of DES concentration of MIPS-coated UCNPs and NIPScoated UCNPS. 
where $F_{0}$ is the initial fluorescence intensity, $F$ is the fluorescence intensity in the presence of DES, $K_{\mathrm{SV}}\left(\mathrm{mL} \mathrm{ng}^{-1}\right)$ is the quenching constant, and $C_{\mathrm{q}}\left(\mu \mathrm{g} \mathrm{mL} \mathrm{mL}^{-1}\right)$ is the concentration of DES. The fluorescence intensity at different concentrations of DES is shown in Fig. 4a. The regression equation is $F_{0} / F=$ $0.00087 C_{\mathrm{q}}+0.0415$, with a correlation coefficient of 0.9989 . The detection limit was found to be $12.8 \mathrm{ng} \mathrm{mL}^{-1}$ by calculating ( $\mathrm{S} / \mathrm{N}$ $=3$ ).

Upon comparing Fig. 4a with Fig. 4b, it could be observed that both MIP-coated UCNPs and NIP-coated UCNPs showed a response to DES. The ratio between the $K_{\mathrm{SV}}$ of MIP-coated UCNPs and the $K_{\mathrm{SV}}$ of NIP-coated UCNPs was 6.87 , as obtained from Fig. 4c, which indicated that the as-synthetized materials had a good specificity towards the target molecule. The main reason for the enhanced response was that MIPcoated UCNPs could match the target molecules much better through the recognition sites. Compared with the results obtained by other procedures for the detection of DES, shown in Table S2, $\uparrow$ this procedure was reproducible and without a complicated operation process.

\section{Selectivity of the MIP-coated UCNPs}

When DES was removed after elution, specific recognition sites were formed on the imprinted cavities, which could selectively rebind DES. To verify the specificity of this sensor, three structural analogues of DES (DINE, HEX, and BPA) and a functional analogue of DES $\left(\mathrm{E}_{2}\right)$ were selected (structures shown in Fig. S3 $\dagger$ ). As shown in Fig. 5, MIP-coated UCNPs showed a good specificity towards DES, and the quenching with the analogues was due to nonspecific adsorption on the imprinted cavities. The $K_{\text {SV }}$ value of MIP-coated UCNPs for DES was much larger than that for the structural and functional analogues because of the shape, location, and the size of the binding cavities. In summary, these results confirmed that this sensor has a high selectivity towards DES.

\section{Fluorescence repeatability and stability of the MIP-coated UCNPs}

The repeatability of the sensor was evaluated by the following method: $5 \mathrm{mg}$ of MIP-coated UCNPs and $2 \mathrm{~mL}$ of DES

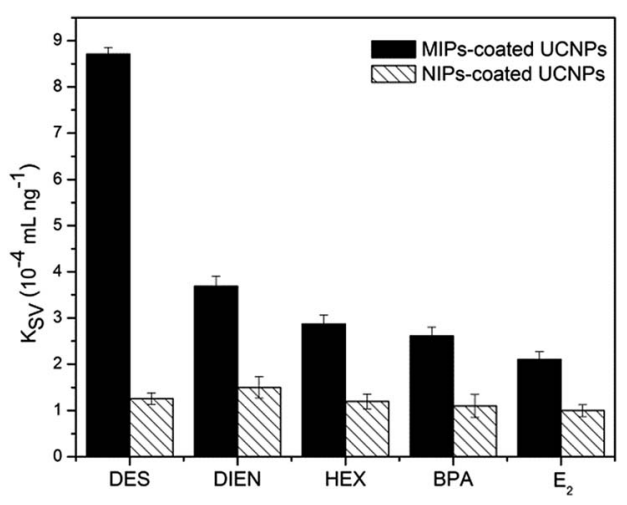

Fig. 5 The Stern-Volmer quenching constants of different analogues with MIPs-coated UCNPs and NIPs-coated UCNPs.
Table 1 Recovery of DES at different concentrations in milk samples $(n=5)$

\begin{tabular}{llccc}
\hline Sample & $\begin{array}{l}\text { Spiking levels } \\
\left(\mathrm{ng} \mathrm{mL} \mathrm{mL}^{-1}\right)\end{array}$ & $\begin{array}{l}\text { Amount measured } \\
\left(\mathrm{ng} \mathrm{mL}^{-1}\right)\end{array}$ & $\begin{array}{l}\text { Recovery } \\
(\%)\end{array}$ & $\begin{array}{l}\text { RSD } \\
(\%)\end{array}$ \\
\hline 1 & 60 & 54.15 & 90.3 & 2.3 \\
2 & 120 & 127.07 & 105.9 & 1.5 \\
3 & 240 & 235.39 & 98.1 & 1.9 \\
4 & 480 & 503.73 & 104.9 & 1.7 \\
5 & 960 & 1034.57 & 107.8 & 2.7
\end{tabular}

acetonitrile solution with the concentration of $100 \mu \mathrm{L} \mathrm{mL} \mathrm{m}^{-1}$ were added to a $5 \mathrm{~mL}$ Eppendorf tube and stirred for $4 \mathrm{~h}$ at room temperature. The supernatant was analyzed by UV-vis spectrophotometry and MIP-coated UCNPs were obtained by centrifugation and washed three times with methanol : HAc $(9: 1, \mathrm{v} / \mathrm{v})$. The abovementioned steps were repeated 7 times. As shown in Fig. S4a, $\dagger$ the MIP-coated UCNPs could still reach $90 \%$ of the adsorption capacity after 7 cycles. This result demonstrated that MIP-coated UCNPs could be recycled, thus reducing testing costs.

To investigate the analytical stability of the MIPs-coated UCNPs, the following experiment was designed. The repeated test of the fluorescent response intensity was performed every $10 \mathrm{~min}$. The results, as shown in Fig. $\mathrm{S} 4 \mathrm{~b}, \dagger$ indicated that the fluorescence response intensity of MIP-coated UCNPs was stable within $60 \mathrm{~min}$. Moreover, the repeated test of the fluorescent response intensity was also performed every day. On the seventh day, MIP-coated UCNPs still retained 95\% of its initial response, as shown in Fig. S4c. $\uparrow$ This result indicated that MIPcoated UCNPs could be stable for at least 7 days at room temperature. The excellent fluorescence stability of this sensor showed a promising application for the on-field detection of DES.

\section{Applications in the analysis of a milk sample}

To further demonstrate the applicability of MIP-coated UCNPs in a real sample, the concentration of DES was determined in milk. The concentrations of spiked DES in milk samples were

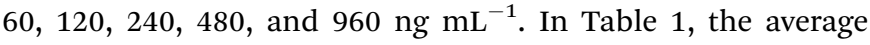
recoveries were found to be in the range from 90.3 to $107.8 \%$ with RSDs from 1.5 to $2.7 \%$. These results indicated that the developed method was accurate and the pre-processing procedure was simple, demonstrating that the method could be applied in real sample monitoring.

\section{Conclusion}

In summary, a novel procedure for detecting diethylstilbestrol was successfully developed on the basis of MIP-coated UCNPs, which combined the fluorescence properties of $\left.\mathrm{NaYF}_{4}: \mathrm{Yb}\right] \mathrm{r}$ upconversion nanoparticles with the selectivity of a molecularly imprinted polymer. We deposited a silicon shell and modified the double bond on the surface of the UCNPs by a one-step process and prepared the imprinted layer on the exterior of 
the UCNPs by the surface graft molecular imprinting method. The synthetic procedure was simple and easy to control. The label-free sensor was considered to have an excellent fluorescence, outstanding stability, good repeatability, high selectivity, and a wide detection range, and could be applied for the analysis of real samples. It was suggested that the simple and reliable preparation strategy of MIP-coated UCNPs could be a great potential method for customizing sensing materials according to the hazardous compounds found in food products.

\section{Acknowledgements}

This work was supported by the National Natural Science Foundation of China (No. 21477162, No. AWS15J006), the Science and Technology Planning Projects of Tianjin (No. 14ZCZDSF00021), the National Key Scientific Instrument and Equipment Development Project (No. 2013YQ14037106).

\section{Notes and references}

1 J. Wang, H. Ye, Z. Jiang, N. Chen and J. Huang, Anal. Chim. Acta, 2004, 508, 171-176.

2 S. Zhang, B. Du, H. Li, X. D. Xin, H. M. Ma, D. Wu, L. G. Yan and Q. Wei, Biosens. Bioelectron., 2014, 52, 225-231.

3 D. Ganmaa and A. Sato, Med. Hypotheses, 2005, 65, 10281037.

4 D. Ganmaa, H. Tezuka, D. Enkhmaa, K. Hoshi and A. Sato, Int. J. Cancer, 2006, 118, 2363-2365.

5 L. Q. Qin, P. Y. Wang, T. Kaneko, K. Hoshi and A. Sato, Med. Hypotheses, 2004, 62, 133-142.

6 Q. L. Zhang, J. Li, T. T. Ma and Z. T. Zhang, Food Chem., 2008, 111, 498-502.

7 W. Yan, Y. Li, L. Zhao and J. M. Lin, J. Chromatogr. A, 2009, 1216, 7539-7545.

8 K. McMartin, K. Kennedy, P. Greenspan, S. Alam, P. Greiner and J. Yam, J. Environ. Pathol. Toxicol., 1978, 1, 279.

9 J. Król, W. Pobłocki, T. Bockenheimer and P. Hliwa, Aquacult. Int., 2014, 22, 53-62.

10 C. L. Ke, Z. H. Wang, J. L. Gan, Y. G. Gu, K. Huang, L. D. Li and Q. Lin, RSC Adv., 2014, 4, 2355-2359.

11 G. Kaklamanos, G. Theodoridis and T. Dabalis, J. Chromatogr. A, 2009, 1216, 8072-8079.
12 F. Guo, Q. Liu, G. B. Qu, S. J. Song, J. T. Sun, J. B. Shi and G. B. Jiang, J. Chromatogr. A, 2013, 1281, 9-18.

13 Z. D. Yan, P. Xiong, N. Gan, J. L. He, N. B. Long, F. T. Hu and T. H. Li, J. Electroanal. Chem., 2015, 736, 30-37.

14 J. S. Tang, L. Xiang, F. Zhao, F. K. Pan, S. Y. Wang and X. F. Zhan, Anal. Lett., 2015, 48, 796-808.

15 D. Mukunzi, B. N. Tochi, J. Isanga, L. Q. Liu, H. Kuang and

C. L. Xu, Food Agric. Immunol., 2016, 27, 855-869.

16 Y. Chen, Z. Q. Wang, Z. H. Wang, S. S. Tang, Y. Zhu and

X. L. Xiao, J. Agric. Food. Chem., 2008, 56, 2944-2952.

17 L. Cheng, C. Wang and Z. Liu, Nanoscale, 2013, 5, 23-37.

18 L. Wang and Y. Li, Chem. Mater., 2007, 19, 727-734.

19 W. Zhang, X. W. He, Y. Chen, W. Y. Li and Y. K. Zhang, Biosens. Bioelectron., 2012, 31, 84-89.

20 D. Y. Li, X. W. He, Y. Chen, W. Y. Li and Y. K. Zhang, ACS Appl. Mater. Interfaces, 2013, 5, 12609-12616.

21 F. Wang and X. Liu, Chem. Soc. Rev., 2009, 38, 976-989.

22 K. Haupt, Anal. Chem., 2003, 75, 376A-383A.

23 K. Haupt and K. Mosbach, Chem. Rev., 2000, 100, 2495-2504.

24 A. Poma, A. Guerreiro, M. J. Whitcombe, E. V. Piletska, A. P. Turner and S. A. Piletsky, Adv. Funct. Mater., 2013, 23, 2821-2827.

25 J. Wackerlig and P. A. Lieberzeit, Sens. Actuators, B, 2015, 207, 144-157.

26 Y. Tang, Z. Gao, S. Wang, X. Gao, J. Gao, Y. Ma, X. Liu and J. Li, Biosens. Bioelectron., 2015, 71, 44-50.

27 J. Tian, J. Bai, Y. Peng, Z. Qie, Y. Zhao, B. Ning, X. Dan and Z. X. Gao, Analyst, 2015, 140, 5301-5307.

28 Y. Cen, J. Tang, X. J. Kong, S. Wu, J. Yuan and R. Q. Yu, Nanoscale, 2015, 7, 13951-13957.

29 K. Stalder and W. Stöber, Nature, 1965, 207, 874-875.

30 S. Wu, N. Duan, X. Ma, Y. Xia, Y. Yu, Z. Wang and H. Wang, Chem. Commun., 2012, 48, 4866-4868.

31 X. Zhang, Y. Peng, J. Bai, B. Ning, S. Sun, X. Hong, Y. Y. Liu, Y. Liu and Z. X. Gao, Sens. Actuators, B, 2014, 200, 69-75.

32 T. Guo, Q. Deng, G. Fang, C. Liu, X. Huang and S. Wang, Biosens. Bioelectron., 2015, 74, 498-503.

33 X. Zhou, P. Ma, A. Wang, C. Yu, T. Qian, S. Wu and J. Shen, Biosens. Bioelectron., 2015, 64, 404-410. 